Website: http://revistas.lamolina.edu.pe/index.php/acu/index

(C) Universidad Nacional Agraria La Molina, Lima - Perú

\title{
Producción y características morfologicas de genotipos de pasto elefante enano (Pennisetum purpureum, Schum) sobre pastoreo
}

\author{
Production and morphological characteristics of genotypes of dwarfish elephant grass \\ (Pennisetum purpureum, Schum) under rotational grazing
}

\author{
Lizbeth Collazos Paucar ${ }^{1 *}$; Hernán Maldonado Vásquez²; José Coelho da Silva²; Geraldo de Amaral Gravina²; \\ Lucival de Souza Jr.2; Tânia de França Padilha² \\ * Autor de correspondencia
}

\section{Resumen}

El objetivo de este trabajo fue evaluar la producción, y las características morfológicas de tres genotipos de pasto elefante enano Pennisetum purpureum, Schum: (CNPGL 92198-7, CNPGL 94-34-3 y cv. Mott) sobre el pastoreo. El experimento fue conducido en el Sector de Forragicultura y Nutrición de Rumiantes (LZNA/CCTA) de la Universidad Estadual del Norte Fluminense Darcy Ribeiro, Campos de los Goytacazes, RJ, Brasil. El análisis estadístico fué bloques completos al azar, con parcelas subdivididas, con tres repeticiones. Las parcelas consistieron de tres genotipos de pasto elefante enano, mientras que las subparcelas fueron representadas por cuatro frecuencias de pastoreo: (30 y 45 días de descanso en época de lluvias y seca respectivamente), cuando el dosel forrajero alcanzaba 90, 95 y 100 \% de interceptación de luz. Se verificó efecto del factor época sobre la producción, altura, cobertura y tasa de persistencia, con medias más altas en época de lluvias $(\mathrm{P}<0,01)$, en comparación con la época seca. El efecto de época del año sobre las variables analizadas confirma el comportamiento estacional del pasto elefante enano.

Palabras clave: cv. Mott; interceptación luminosa; pastoreo rotacional; persistencia.

\begin{abstract}
Abstrac
The objective of this work was to evaluate the production and morphological characteristics of dwarf elephant grass Pennisetum purpureum, Schum: (CNPGL 92198-7, CNPGL 94-343, cv. Mott) subjected to a grazing system. The experiment was conducted in the Sector of Forragiculture and Nutrition of Ruminants (LZNA / CCTA) of the State University of North Fluminense Darcy Ribeiro, Campos dos Goytacazes, Rio de Janeiro. The statistical analysis was randomized blocks with subdivided plots, with three repetitions. The parcels were composed by the three genotypes of dwarfish elephant grass, while the sub parcels were represented by four grazing frequencies: 30 and 45 days of resting period, respectively, for the raining and dry seasons, and when the forage canopy reached 90, 95 and $100 \%$ of luminous interception. The effect of the season of the year was measured on height of the sward, soil covering, and the persistence rate of the tillers, with higher averages for the rainy season $(\mathrm{P}<0.01)$. The genotypes with larger height of the forage canopy in the pre grazing were the cv. Mott and the genotype CNPGL 92198-7, while the genotype CNPGL 9434-3 presented the largest tillering rate. The effect of the season of the year on the analyzed variables confirms the behavior sharply seasonal of the dwarfish elephant grass.
\end{abstract}

Keywords: cv. Mott; luminous interception; rotational grazing; tiller.

\section{Introducción}

Las especies de clima tropical $\left(\mathrm{C}_{4}\right)$ superan las de clima templado $\left(\mathrm{C}_{3}\right)$ en cuanto a la capacidad fotosintética, tasa de crecimiento, eficiencia en el uso del agua, nutriente e interceptación de la luz (Mott., 1984). En este contexto, existe demanda por forrajeras con elevado potencial productivo y calidad nutricional para mantener elevada productividad animal durante todo el año. El potencial productivo del pasto-elefante (Pennisetum purpureum Schum.) es determinado por varios factores, como: genotipo, intervalos y altura de corte, disponibilidad de nutrientes, agua, luz y temperatura (Jacques, 1990; Lavezzo, 1992), es cultivado en todas las regiones tropicales y subtropicales del mundo, debido a su rápido crecimiento, mayor capacidad de acumulación de materia seca (MS), calidad nutricional, aceptabilidad, vigor y persistencia (Pereira et al., 2001). Entre los factores que afectan el flujo de biomasa de una gramínea forrajera, las yemas es el que ejerce mayor influencia sobre la acumulación de forraje. La aparición y el crecimiento de hojas posibilitan la restauración del

\footnotetext{
${ }^{1}$ Departamento Académico de Producción Animal, Facultad de Zootecnia, Universidad Nacional Agraria La Molina, Apartado postal 12-056- La Molina, Lima, Perú. Email: Lcollazos@lamolina.edu.pe

${ }^{2}$ Laboratório de Zootecnia e Nutrición Animal (LZNA), Centro de Ciencias e Tecnologias Agropecuárias (CCTA), UENF/Campos dos Goytacazes, Rio de Janeiro, Brasil.
} 
área foliar de las gramíneas forrajeras después del corte o pastoreo y ayudan en el mantenimiento de la producción de forraje y perennidad del pastoreo (Cunha et al., 2010). El desempeño animal varía debido a la presión de pastoreo, por su efecto sobre las características estructurales de masa, altura, porcentaje de material senescente del forraje y consumo de forraje (Almeida et al., 2002). Con la comprensión del comportamiento de la planta a las condiciones edafoclimáticas de su ambiente, es posible recomendar prácticas de manejo basadas en la condición del pasto (altura, masa de forraje, índice de área foliar, interceptación de luz), para maximizar la productividad forrajera y la eficiencia de la cosecha del forraje por el animal en pastoreo. El objetivo de este trabajo fue evaluar la producción y características morfológicas de tres genotipos de pasto elefante enano Pennisetum purpureum Schum (CNPGL 92198-7, CNPGL 94-343 y cv. Mott) en pastoreo.

\section{Materiales y métodos}

\section{Área Experimental}

El experimento fue realizado en el Sector de Forraje y Nutrición de Ruminantes del Laboratorio de Zootecnia y Nutrición Animal (LZNA), de la Universidad Estatal del Norte Fluminense (UENF), en Campos dos Goytacazes (RJ). Su clima es tropical caliente y húmedo, con un período seco en invierno y lluvioso en el verano. El suelo del área experimental se clasifica como Latosolo amarillo de textura arenosa. La fertilización se realizó de acuerdo con los resultados de análisis de suelo, el área experimental de 2,0 ha se dividio en cuatro potreros de $500 \mathrm{~m}^{2}$.

\section{Diseño experimental y manejo de animales}

El delineamiento experimental fue el de bloques completos al azar, en parcelas subdivididas, con tres repeticiones, 12 tratamientos y 36 unidades experimentales. Se utilizaron 12 bovinos mestizos (holandés x cebú), con peso promedio de $300 \mathrm{~kg}$. En cada tratamiento, se utilizaron tres animales con tres días de ocupación. Después del tercer día de pastoreo se realizó corte de uniformización para establecer la altura del pasto en $40 \mathrm{~cm}$. Para la estimación de la cobertura (\%), área libre (\%) y materia inerte (\%) se realizaron 10 lecturas o estimaciones visuales a cada ciclo de pastoreo.

\section{Tratamientos}

Las parcelas fuerón representadas por tres genotipos de pasto elefante enano Pennisetum purpureum Schum (CNPGL 92198-7, CNPGL 94-343 y cv. Mott) mientras que las subparcelas correspondieron a cuatro frecuencias de pastoreo: (30 y 45 días de descanso en época de lluvias y seca, respectivamente) y a tres condiciones de pastoreo representadas por niveles de Interceptación de luz (IL) de 90,95 y $100 \%$.

\section{Interceptación de luz}

Las lecturas de altura en la condición de pre-pastoreo se realizaron cuando los potreros alcanzaron los niveles de
90, 95 y 100 \% de interceptación de luz; y 30 o 45 días de descanso. Determinación de los niveles de interceptación de luz por el dosel forrajero a través de mediciones por la mañana (entre 10:00 y 12:00 h), con cielo claro, midiendo la intensidad de luz por encima del forraje y al nivel del suelo, utilizando un analizador AccuPAR Linear PAR/ LAI, Modelo PAR-80 (DECAGON Devices). Se realizaron lecturas por unidad experimental según recomendaciones (Carnavelli et al., 2003). En cada estación se realizaron 3 lecturas.

\section{Composición Botánica}

La composición botánica de los genotipos fue realizada mediante lecturas de estimaciones visuales (10 lecturas) para determinar los porcentajes de cobertura, área libre, y materia seca inerte. La altura de la planta fue determinada a partir del nivel del suelo hasta la altura de la curvatura de las hojas superiores completamente expandidas, utilizando una regla graduada en centímetros, midiendo 20 puntos aleatorios por unidad experimental (Cunha et al. 2010). Las lecturas de altura en la condición de pre-pastoreo, se realizaron cuando los potreros alcanzaron los niveles de 90, 95 y $100 \%$ de interceptación de luz, y 30 o 45 días de descanso.

\section{Disponibilidad forrajera}

La producción de biomasa de pre-pastoreo se midió con un marco de $1 \mathrm{~m}^{2}$, lanzado al azar tres veces por unidad experimental. Los cuadrados fueron colocados aleatoriamente en puntos representativos de la altura media del dosel forrajero de cada potrero y el forraje contenido en el interior del cuadro fue cortado a nivel del suelo. Para la evaluación de los componentes morfológicos del forraje se retiró una alícuota para la determinación de la MS de pre-pastoreo. Esta alícuota fue pesada y fraccionada en lámina foliar, tallo + vaina y material senescente. Estos componentes fueron evaluados y pesados por separado para determinar el contenido y la disponibilidad de MS por componente de la planta. Posteriormente los componentes fueron deshidratados en una estufa a $55^{\circ} \mathbf{C}$ por 72 horas hasta alcanzar peso constante, el cual fue procesado molido en un molino Willey con cribas de $1 \mathrm{~mm}$. Los valores obtenidos se convirtieron en $\mathrm{kg} \mathrm{MS} / \mathrm{ha}$ y los componentes morfológicos expresados como proporción (\%) de la masa de forraje.

\section{Diseño experimental y análisis estadístico}

El diseño experimental fue el de bloques completos al azar, en parcelas subdivididas, con tres repeticiones, Las parcelas consistían en tres genotipos de pasto elefante enano, en las sub parcelas los niveles de interceptación de luz $(90,95,100 \%)$ y período de descanso fijo 30 a 45 días, respectivamente para época de lluvias y seca. Las diversas variables fueron sometidos al análisis de varianza mediante programa estadístico (versión 9.4 SAS Institute Inc., Cary, NC, EUA, 2013), siendo las medias comparadas por el teste de Tukey al $5 \%$ de significancia. 


\section{Resultados y discusión}

En la Tabla 1 se observan las diferencias entre épocas, en época de lluvias los valores medios fueron de 3.536,75, $3.821,91 \mathrm{~kg} / \mathrm{ha}$ y 1,56 , respectivamente, para las variables PMSLF, PMSLFCB y relación lámina foliar/tallo, siendo mayores producciones de PMSLF en relación PMSTB, la lámina foliar constituye una característica deseable, por estar relacionada con la calidad nutricional y el consumo.

Tabla 1. Valores medios de producción de MS de lámina foliar (PMSLF/kg/ha), producción de MS de tallo + vaina (PMSTB $/ \mathrm{kg} / \mathrm{ha}$ ), producción de MS de lámina foliar y tallo + vaina (PMSLFTB/kg/ha), producción de MS senescente (PMSs/kg/ha) y relación lámina foliar y tallo (RLF/T), evaluados durante las épocas de lluvias y seca de pasto elefante enano (Pennisetum purpureum Schum)

\begin{tabular}{cccccc}
\hline Épocas & PMSLF & PMSTB & PMSLFTB & PMSs & R LF/T \\
\hline Águas & $3536,75^{\mathrm{a}}$ & $285,17^{\mathrm{b}}$ & $3821,91^{\mathrm{a}}$ & $262,54^{\mathrm{b}}$ & $1,56^{\mathrm{a}}$ \\
Seca & $2107,54^{\mathrm{b}}$ & $547,02^{\mathrm{a}}$ & $2651,25^{\mathrm{b}}$ & $369,14^{\mathrm{a}}$ & $1,40^{\mathrm{b}}$ \\
\hline
\end{tabular}

Diferentes letras dentro de una misma columna para cada época indican diferencias estadísticas, Tukey $(\mathrm{p}<0.05)$

Queiroz Filho et al. (1998), evaluando genotipos de pasto elefante encontraron para cv. Cameroon, 1,15 en relación lámina foliar/tallo. El resultado encontrado en este trabajo fue mayor en relación de Según Da Silva \& Nascimento (2006), el crecimiento de las yemas interfiere en la estructura de dosel forrajero y en el equilibrio de los procesos de interceptación de luz. Estos comportamientos pueden estar relacionados con la fotosíntesis como fuente precursora del crecimiento vegetativo, con acumulación de MS siendo determinado por la interceptación de luz en el dosel forrajero (Carnevalli et al., 2006). La distribución de la luz dentro de la comunidad vegetal tiene mayor importancia que la producción de MS (Da Silva y Nascimento Júnior, 2007). Se observo que el factor época influenció la altura de los forrajes en condiciones de prepastoreo, la cobertura y tasa de persistencia de yemas $(\mathrm{P}>$ 0,05 ), con valores más elevados en época de lluvias (Tabla 2).

Tabla 2. Los valores medios de altura pre-pastoreo, cobertura, tasa de persistencia de yemas (TPP) y MS inerte (MSI) de genotipos de pasto elefante enano (CNPGL 92198-7, CNPGL 9434-3, cv Mott) durante la época de lluvias y seca

$\begin{array}{ccccc}\text { Épocas } & \text { Altura }(\mathrm{cm}) & \text { Cobertura }(\%) & \mathrm{TPP} / \mathrm{m}^{2} & \mathrm{MSI}(\%) \\ \text { Lluvias } & 108 \mathrm{~A} & 74,59 \mathrm{~A} & 1,03 \mathrm{~A} & 24,14 \mathrm{~B} \\ \text { Seca } & 64 \mathrm{~B} & 65,77 \mathrm{~B} & 0,85 \mathrm{~B} & 32,92 \mathrm{~A} \\ \begin{array}{c}\text { Diferentes letras dentro de una misma columna para cada época indican } \\ \text { diferencias estadísticas, Tukey }(\mathrm{p}<0.05)\end{array}\end{array}$
diferencias estadísticas, Tukey $(\mathrm{p}<0.05)$

La aparición de yemas es controlada por el genotipo de cada especie forrajera, y en este trabajo, el genotipo CNPGL 9434-3 presentó mayor media para tasa de aparición de yemas. La frecuencia de pastoreo basadas en los niveles de 90, 95 y $100 \%$ de interceptación de luz no comprometieron la estabilización o equilibrio del número de yemas, no hubo efecto de IL para tasa de aparición y mortalidad de yemas entre genotipos. El cultivar Mott y el genotipo CNPGL 92198-7 presentaron promedios más altos de altura pre-pastoreo (Tabla 3).

Tabla 3. Valores medios de altura pre-pastoreo y tasa de aparición de yemas (TAY) de tres genotipos de pastoelefante enano (CNPGL 92198-7, CNPGL 9434-3, cv Mott)

\begin{tabular}{ccc}
\hline Genotipos & Altura $(\mathrm{cm})$ & $\mathrm{TAP} / \mathrm{m}^{2}$ \\
\hline CNPGL 92198-7 & $90 \mathrm{~A}$ & $0,69 \mathrm{~B}$ \\
CNPGL 9434-3 & $77 \mathrm{~B}$ & $0,84 \mathrm{~A}$ \\
Cultivar Mott & $91,70 \mathrm{~A}$ & $0,72 \mathrm{~B}$ \\
\hline
\end{tabular}

Diferentes letras dentro de una misma columna para cada época indican diferencias estadísticas, Tukey $(\mathrm{p}<0.05)$

En la Tabla 3 se observa diferencias de altura de los genotipos, siendo que cv. Mott el que alcanzo mayor altura (91,70 cm) seguido del genotipo CNPGL 92198-7 $(90 \mathrm{~cm})$; los cuales presentaron mayores valores medios en relación al genotipo CNPGL 9434-3 con (77 cm). Se observó para genotipo CNPGL 9434-3 mayor tasa de aparición de yemas basales de $\left(0,84 / \mathrm{m}^{2}\right)$ en relación a cv. Mott $\left(0,72 / \mathrm{m}^{2}\right)$ y el genotipo CNPGL $92198-7\left(0,69 / \mathrm{m}^{2}\right)$. Según (Gomide \& Paciullo, 2012), la producción de yemas es controlada por el patrón de aparición de hojas y persistencia de yemas, siendo afectada por su manejo e intervalo de pastoreo. Carvalho et al (2007), mencionan que el crecimiento de yemas de gramíneas forrajeras es una de las características más importantes para el aumento de la productividad de las plantas, pero puede ser influenciada por el sistema de manejo en pastoreo.

\section{Conclusiones}

Los genótipos de pasto elefante presentaron mejores comportamientos en cuanto a altura, cobertura de gramíneas, tasa de persistencia de yemas $/ \mathrm{m}^{2}$, producción de lámina foliar, relación lámina foliar/tallo, en época de lluvias en relación la seca.

\section{Literatura citada}

Almeida, R.G.; Nascimento Jr. D.; Euclides, V. P.B. et al. 2002. Produção animal en pastos consorciados sob três taxas de lotação, no cerrado. Revista Brasileira de Zootecnia. 31 (2): 852-857.

Carnavelli, R. A. et al., 2003. Dinâmica da rebrotação de pastos de capim-Mombaça submetidos a regimes de desfolhação intermitente. Tese de Doutorado em Agronomia- Ciência Animal e Pastagens, Piracicaba, ESALQ.

Carnevalli, R.A. et al. 2006. Herbage production and grazing losses in Panicum maximum cv. Mombaça under four grazing managements. Tropical Grasslands, 40: 165176 . 
Cunha B A L. et al. 2010. Effects of two post grazing heights on morphogenic and structural characteristics of Guinea grass under rotational grazing. Tropical Grasslands, 44(2), 253-259.

Carvalho, C.A.B. de; Rossielo, R.O.P.; Paciullo, D.S.C.; Sbrissia, A.F.; Deresz, F. 2007. Classes de perfilhos na composição do índice de área foliar em pastos de capimelefante. Pesquisa Agropecuária Brasileira, 42:557563.

Da Silva \& Nascimento JR 2006 Ecofisiologia de plantas forrageiras. In: PEREIRA, O.G. OBEID, J.A.; NASCIMENTO Jr., D.; FONSECA, D.M. (Eds.). Simpósio sobre manejo estratégico da pastagem, III, Viçosa, 2006. Anais. Viçosa: 430p.

Da Silva, S.C.; Nascimento Junior, D. 2007. Avanços na pesquisa com plantas forrageiras tropicais em pastagens: características morfofisiológicas e manejo do pastejo. Revista Brasileira de Zootecnia, 36: 21138.

Jacques, A.V.A. 1990. Fisiologia do crescimento do capimelefante (Pennisetum purpureum

Schum.). In: SIMPÓSIO SOBRE CAPIM-ELEFANTE, Juiz de Fora, MG, 1990. Anais. Coronel Pacheco: EMBRAPA-CNPGL, 23-33P.

Lavezzo, W. 1992. Ensilagem do capim-Elefante. Anais do Simpósio Sobre Manejo de Pastagem, 10, 169-275P.

Mott, G.O. 1984. Carrying capacity and live weight gains from dwarf elephant-grass. In: FLORIDA BEEF CATTLE SHORT COURSE PRACTICE, 1., 1984, Gainesville, Anais. Gainesville, 111-114P.

Pereira, A.V. et al. 2001. Melhoramento de forrageiras tropicais. In: Nass, L. L.; Valois, A.C.C.; Melo, I. S.; Valadares-Inglis, M. C. Recursos genéticos e melhoramento. Rondonópolis: Fundação Mato Grosso, 549-602.

Queiroz Filho, J.1., Silva, D.S., Nascimento, I.S. et al. 1998. Produção de matéria seca e qualidade de cultivares de capim-elefante (Pennisetum purpureum, Schum.). R. Bras. Zootec., 27(2):262-266.

SAS. 2013. User's Guide. Statistics. Version 9.4 ed. Cary. NC: SAS Inst. 